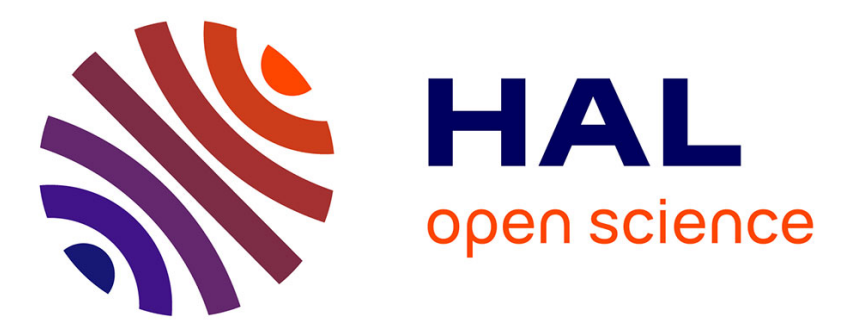

\title{
Greffe courte quatre brins dans la reconstruction du ligament croisé antérieur. Le droit interne 4 brins est-il une solution fiable? Étude biomécanique
}

Antoine Combes, Laure-Lise Gras

\section{- To cite this version:}

Antoine Combes, Laure-Lise Gras. Greffe courte quatre brins dans la reconstruction du ligament croisé antérieur. Le droit interne 4 brins est-il une solution fiable? Étude biomécanique. SFA 2016

- Congrès annuel de la Société Francophone d'Arthroscopie, Nov 2016, PARIS, France. pp.S275, 10.1016/j.rcot.2016.10.012 . hal-01844494

\author{
HAL Id: hal-01844494 \\ https://hal.science/hal-01844494
}

Submitted on 19 Jul 2018

HAL is a multi-disciplinary open access archive for the deposit and dissemination of scientific research documents, whether they are published or not. The documents may come from teaching and research institutions in France or abroad, or from public or private research centers.
L'archive ouverte pluridisciplinaire HAL, est destinée au dépôt et à la diffusion de documents scientifiques de niveau recherche, publiés ou non, émanant des établissements d'enseignement et de recherche français ou étrangers, des laboratoires publics ou privés. 
https://doi.org/10.1016/j.rcot.2016.10.012

Revue de Chirurgie Orthopédique et Traumatologique

Travaux de la Société Francophone d'Arthroscopie

\section{Communication particulière}

Greffe courte quatre brins dans la reconstruction du ligament croisé antérieur. Le Droit interne 4 brins est il une solution fiable ? Etude biomécanique

Short hamstring four bundle graft in anterior cruciate ligament reconstruction. Is the gracilis a reliable solution? A biomechanical study

Antoine Combes ${ }^{* a}$, Laure-Lise Gras ${ }^{b, c, d}$

* Premier auteur, auteur orateur

a. Centre de chirurgie orthopédique Fleming, F38300, Bourgoin Jallieu ;

b. Université de Lyon, F-69622, Lyon, France ;

c. Université Claude Bernard Lyon 1, Villeurbanne ;

d. IFSTTAR, UMR_T9406, LBMC Laboratoire de Biomécanique et Mécanique des Chocs, F69675, Bron ;

Mots clés: Reconstruction du ligament croisé antérieur, droit interne, demi-tendineux, expérimentation in vitro, propriétés mécaniques, faufilage, greffe quatre brins

Introduction: Dans le cadre de la reconstruction du ligament croisé antérieur, la greffe au demitendineux quatre brins (DT4) est devenue une solution fréquente et reproductible. L'utilisation d'un seul ischio-jambier ouvre la possibilité d'utiliser l'ischio-jambier restant - le droit-interne (DI) - lors d'un éventuelle reprise chirurgicale.

Matériel et méthodes : Les tendons DI et DT sont prélevés sur six sujets cadavériques embaumés. Des greffes à quatre brins sont réalisées : 12 DI et 12 DT. Chaque greffe est testée selon le même protocole expérimental. La greffe est placée sur une machine de traction. Une pré-charge de $1 \mathrm{~N}$ lui est appliquée puis l'effort est amené à $50 \mathrm{~N}$ et maintenu pendant 30s. Mille cycles de chargementdéchargement d'amplitude $200 \mathrm{~N}$ sont ensuite appliqués à la fréquence de $1 \mathrm{~Hz}$. Enfin, la greffe est allongée jusqu'à rupture à la vitesse de $20 \mathrm{~mm} / \mathrm{min}$. L'évolution de l'allongement de la greffe et de la raideur au cours des cycles, la raideur avant rupture et la charge à rupture sont étudiées et comparées avec une étude statistique.

Résultats : Au $1000^{\text {ème }}$ cycle, l'allongement des greffes DI $(4.3 \mathrm{~mm})$ est supérieur à celui des greffes DT $(3.3 \mathrm{~mm})$, mais cette différence n'est pas statistiquement significative. La raideur augmente au cours des cycles. Il existe une différence significative entre la raideur des greffes DI (230N/mm) et DT $(266 \mathrm{~N} / \mathrm{mm})$ au $1000^{\mathrm{ème}}$ cycle ( $\mathrm{p}$-value $\left.<0.02\right)$. La différence est significative entre la raideur avant rupture des greffes DI $(290 \mathrm{~N} / \mathrm{mm})$ et des greffes DT $(340 \mathrm{~N} / \mathrm{mm})(p$-value $<0.02)$. La charge à rupture $n^{\prime}$ est pas statistiquement différente entre les greffes DI (882N) et DT (1094N).

Discussion : L'allongement de la greffe DI est augmenté par rapport au DT. La différence n'est pas significative, peut-être secondaire au faible nombre de sujets. L'allongement des deux types de greffes est conséquent mais comparable aux données de la littérature. Cet allongement est probablement plus faible in vivo puisque la tension de serrage des systèmes de fixation des greffes courtes (bouton ou le couple bandelette-vis) est proche de $150 \mathrm{~N}$ et donc bien supérieur aux 50N utilisés dans cette étude. 
Conclusion : La greffe avec le droit interne semble présenter des qualités biomécaniques proches de celles avec le demi-tendineux. Le droit interne pourrait donc être utilisé lors de reprise de ligamentoplastie. 